\title{
Propiedades Psicométricas de la Versión en Español de la Escala de Supervisión Abusiva en Población Laboral Ecuatoriana
}

\author{
Psychometric Properties of the Spanish Version of the Abusive Supervision Scale in \\ Ecuadorian Working Population
}

\author{
Jaime Moreno $^{1}$, Dora Herrera ${ }^{2}$ y Rafael Gargurevich ${ }^{2}$
}

\begin{abstract}
Resumen
El objetivo de este estudio fue traducir, adaptar y analizar las propiedades psicométricas de la Escala de supervisión abusiva (Tepper, 2000). La supervisión abusiva se define como la manifestación sostenida de conductas hostiles verbales y no verbales que excluyen el contacto físico, por parte del jefe directo hacia el colaborador. Si bien la literatura ha crecido en los últimos años, se constata que casi no hay estudios en Iberoamérica. La escala de 15 ítems se aplicó a dos grupos de trabajadores con un intervalo de seis meses (grupo 1, N=308; grupo 2, N=283). En cada grupo se aplicaron diversas medidas y se hizo una prueba de invarianza multi-grupos. El análisis factorial exploratorio y confirmatorio encontró un factor que explicó el $49 \%$ de la varianza y un alfa ordinal de .95. Las diversas medidas empleadas proporcionaron evidencias de validez convergente y discriminante que fueron consistentes con lo reportado en la literatura. Se discuten los hallazgos y sus implicaciones.
\end{abstract}

Palabras clave: supervisión abusiva, satisfacción laboral, intención de rotación, quemado laboral, propiedades psicométricas

\begin{abstract}
This study aimed to translate, adapt and analyze the psychometric properties of the Abusive Supervision Scale (Tepper, 2000), Spanish version. Abusive supervision is defined as the sustained display of hostile verbal and nonverbal behaviors, excluding physical contact, from the supervisor to his direct reports. Although the literature has grown in recent years, it was found that there are almost no studies in IberoAmerica. The 15-item scale was applied to two groups of workers with a six-month interval (group 1, $\mathrm{N}=308$; group 2, $\mathrm{N}=283$ ). Different measures were applied in each group and a multiple-group invariance test was carried out. An exploratory and confirmatory factor analysis yielded a one factor solution that explained $49 \%$ of the variance and an ordinal alpha of .95 . The different measures showed convergent and discriminant validity evidence that was consistent with what was reported in the literature. The findings and their implications are discussed.
\end{abstract}

Keywords: abusive supervision, job satisfaction, turnover intention, burnout, psychometric properties

\footnotetext{
1 Facultad de Psicología, Pontificia Universidad Católica del Ecuador. Correspondencia a: jamorenov@puce.edu.ec; jmv.smk@gmail.com

${ }_{2}^{2}$ Departamento de Psicología, Pontificia Universidad Católica del Perú.
} 


\section{Introducción}

En los últimos años en el ámbito de las organizaciones de trabajo y en el contexto de la relación jefe - colaborador, se observa el incremento de una serie de investigaciones alrededor de un constructo denominado: supervisión abusiva (SA) (Martinko, Harvey, Brees, \& Mackey, 2013). Tepper (2000) define a la SA como "las percepciones de los subordinados respecto al grado en que los supervisores participan en la manifestación sostenida de conductas hostiles verbales y no verbales, que excluyen el contacto físico" (2000, p. 178). Las conductas percibidas como abusivas por parte de los colaboradores son, por ejemplo: criticar a un empleado en presencia de otras personas, ser desconsiderado y grosero, mentir, responsabilizar al colaborador de ciertas acciones para quedar bien, asumir el crédito sin reconocer al colaborador, resaltar los errores de los subordinados, incumplir las promesas, etc. (Tepper, 2000; 2007).

La SA como constructo es parte de una corriente sobre los llamados estilos negativos o destructivos de liderazgo (Burns, 2017) que inició hacia finales del siglo XX con los llamados "líderes tóxicos" (Whicker, 1996). Estos líderes negativos se caracterizan por la manifestación de estilos interpersonales hostiles que tienen un impacto negativo en el bienestar de los colaboradores y en la afectación de los legítimos intereses de la organización (Krasikova, Green \& LeBreton, 2013). Se han propuestos diversos estilos negativos como el liderazgo destructivo (Krasikova, et al., 2013; Thoroughgood, Tate, Sawyer, \& Jacobs, 2012), el liderazgo narcisista (Neider, 2010), los psicópatas corporativos (Boddy, 2011), la pequeña tiranía (Ashforth, 1994) y el liderazgo tóxico (Burns, 2017; Indradevi, 2016; Pelletier, 2010; Reed, 2004; Schmidt, 2008). Si bien las definiciones de estos constructos varían porque resaltan diversos aspectos, Krasikova, et al. (2013) ofrecen una definición amplia sobre la naturaleza del liderazgo destructivo: "Conducta volitiva de un líder que daña o intenta dañar a la organización y/o sus reportes directos (a) alentando al grupo hacia metas contrarias a los legítimos intereses de la organización y/o (b) empleando un estilo de liderazgo que involucra el uso de métodos dañinos de influencia con los seguidores, independientemente de las justificaciones para tal tipo de actuación" (p. 1310).

La SA por su parte, fue propuesta por Tepper (2000) y es el estilo de liderazgo negativo que posiblemente ha recibido la mayor atención en la literatura como lo evidencian recientes meta análisis (Park, Hoobler, Wu, \& Wilson, 2015; Mackey, Frieder, Brees, \& Martinko, 2015; Martinko, et al., 2013; Zhang \& Bednall, 2015). Sin embargo, en Iberoamérica el tema casi no ha sido investigado pues hasta donde se ha podido indagar, hay apenas un par de estudios que abordan explícitamente el tema (Hernández, Espinoza, \& Aguilar, 2013; Xu, Martinez, Van Hoof, Tews, Torres, \& Farfan, 2015) y en los cuales no se utilizó la escala original desarrollada por Tepper (2000) para la medición del constructo.

\section{Supervisión abusiva: antecedentes y consecuencias}

Según Zhang y Bednall (2015) la mayoría de estudios se han centrado en las consecuencias de la SA demostrando que se asocia de manera negativa con la satisfacción laboral (Martinko et al., 2013; Tepper, 2007), el compromiso organizacional, la satisfacción de vida (Frieder, et al., 2015), las conductas cívicas organizacionales (Liu \& Wang, 2013; Organ, Podsakoff, \& MacKenzie, 2006; Zellars, Tepper, \& Duffy, 2002), la calidad del servicio ofrecida a los clientes (Jian, Kwong, Qiu, Qiang, \& Hong-Kit, 2012), la creatividad en el trabajo (Liu, Liao, \& Loi, 2012), las percepciones de justicia organizacional (Gabler \& Hill, 2015; Park, et al., 2015), el compromiso laboral (Scheuer, Burton, Barber, Finkelstein, \& Parker, 2016) y la calidad de la relación jefe - colaborador (Lian, Ferris \& Brown, 2012a).

La SA se relaciona positivamente con otros estilos disfuncionales de liderazgo como el autoritarismo, la impredecibilidad y el liderazgo narcisista (Popa, Rotarescu, Sulea, \& Albulescu, 2013; Pyc, Meltzer, \& Liu, 2017; Schmidt, 2008), con las conductas contraproducentes y las represalias que adoptan los colaboradores que han sido abusados como el robo, el sabotaje y la baja productividad (Martinko, et al., 2013; Mitchell \& Ambrose, 2007; Wei \& Si, 2013), y también con 
indicadores de malestar psicológico como la intención de rotación (Pradhan \& Jena, 2017; Khattak, Ahmad, Ahmad, \& Manzoor, 2015); el agotamiento emocional (Khattak et al., 2015); la depresión y la ansiedad (Pyc, et al., 2017).

La SA también ha sido vinculada con consecuencias que van más allá del ámbito laboral. Carlson, Ferguson, Hunter \& Whitten (2012) encuentran que la SA se relaciona con el conflicto trabajo - familia a través del agotamiento emocional, hallazgo que también es reforzado por Wu \& Cao (2015). Hoobler \& Brass (2006) mencionan que los empleados que son abusados desplazan la agresión que reciben, hacia su familia. La SA también se ha vinculado con el consumo de alcohol (Bamberger \& Bacharach, 2006).

Sobre los antecedentes y mediadores de la SA, Zhang y Bednall (2015) observan que han sido menos investigados y se relacionan con características del supervisor, del colaborador y factores organizacionales. Sobre este último factor Aryee, Chen, Sun, \& Debrah (2007) hablan del "efecto goteo de la supervisión abusiva" que se inicia en los niveles jerárquicos superiores lo que induce conductas abusivas en los niveles inferiores de mando lo que genera, a su vez, un clima laboral de hostilidad interpersonal (Ambrose, Schminke, \& Mayer, 2013) o "clima de supervisión abusiva" (Priesemuth, Schminke, Ambrose, \& Folger, 2014).

\section{Medición de la supervisión abusiva}

Tepper (2000) sostiene que la SA es una evaluación subjetiva que se mide a partir de las percepciones de los colaboradores y desarrolló una escala de 15 ítems que aplicó inicialmente a una muestra de trabajadores en dos momentos diferentes (primer momento, $\mathrm{N}=712$; segundo momento, $\mathrm{N}=362$ ). Los resultados del análisis factorial exploratorio y confirmatorio revelaron una estructura factorial de un solo factor con muy buenas propiedades psicométricas; un coeficiente alto de consistencia interna $(\alpha=.90)$ y evidencias de validez convergente y discriminante con justicia interaccional $(r=-.53)$; justicia procedimental $(r=$ -.48); justicia distributiva ( $r=-.39)$; satisfacción laboral $(r=-.35)$; satisfacción de vida $(r=-.19)$; compromiso de continuidad $(r=.14)$; compromiso normativo $(r=-.27)$; compromiso afectivo $(r=-.24)$; conflicto trabajo a familia $(r=.22)$; conflicto familia a trabajo $(r=.12)$; depresión $(r=.18)$; ansiedad $\quad(r=.21) \quad \mathrm{y}$ agotamiento emocional $(r=.36)$.

\section{Estudios en Iberoamérica}

Se constata apenas dos estudios directos sobre el tema (Hernández, et al., 2013; Xu, et al., 2015). Esto llama la atención pues como afirman Pando, Aranda, Salazar y Torres (2016) la violencia psicológica en el trabajo y el acoso laboral es una realidad persistente y frecuente en Iberoamérica. Moroni y Dabos (2014) afirman que algunas organizaciones usan deliberadamente el abuso "como forma de controlar a los empleados y lograr resultados o como recurso de adoctrinamiento para promover la sumisión y la obediencia extrema" (p. 392). En suma, falta mucho por conocer sobre los factores y la manera en cómo la violencia y el acoso se manifiestan en las organizaciones de diversos países y culturas (Toro \& Gómez-Rubio, 2016). Por otro lado, es claro que el constructo tiene alta relevancia para el bienestar psicológico de la población laboral y la productividad de las organizaciones constituyendo un auténtico factor de riesgo psicosocial (Fernades \& Pereira, 2016).

Estudiar la SA en organizaciones Iberoamericanas permitirá no solamente conocer la prevalencia de este fenómeno y sus consecuencias y antecedentes, sino también la forma en cómo la SA es moldeada por factores organizacionales y culturales (Lian, Ferris, \& Brown, 2012b). Mackey et. al, (2015), plantean que la percepción de SA está moderada por elementos culturales (valores respecto al logro, la autoridad, el colectivismo, la masculinidad, la distancia al poder, etc.). "Las diferencias culturales son especialmente importantes de considerar en el contexto de las percepciones de supervisión abusiva debido al componente de injusticia del supervisor, que es parte de la supervisión abusiva y que puede tener efectos variados en las actitudes y comportamientos de los colaboradores en diversas culturas" (p. 1943). la investigación de un constructo con un mismo instrumento tiene algunas ventajas; se pueden hacer estudios comparativos y analizar las coincidencias o diferencias en variedad de 
muestras y contextos. Esto, a su vez, favorece un desarrollo teórico más consistente.

Es claro entonces que prácticamente no hay estudios sobre la SA en Iberoamérica y que en los pocos trabajos encontrados no se utilizó la escala desarrollada por Tepper (2000), la cual no ha sido traducida ni adaptada previamente al castellano.

\section{Método}

\section{Participantes}

La escala se aplicó a dos grupos de personas con un intervalo de tiempo de seis meses (grupo 1, $\mathrm{N}=308$; grupo 2, $\mathrm{N}=283$ ). Los participantes tenían al menos dos años de experiencia laboral, se encontraban trabajando al momento de llenar la encuesta y reportaban a un jefe directo desde hace al menos seis meses.

\section{Medidas aplicadas en la primera muestra}

Escala de supervisión abusiva (Tepper, 2000). Tiene 15 ítems que son respondidos con un formato Likert de cinco niveles desde 5 (me hace esto todo el tiempo), hasta 1 (no recuerdo que me haya hecho esto). Tepper (2000) reporta una estructura de un solo factor y un coeficiente Alfa de Cronbach $=.90$. Las evidencias de validez de la escala original se obtuvieron analizando patrones de convergencia y discriminancia en 14 variables diferentes que en su conjunto demostraron una validez de constructo robusta. Ejemplos de ítems: me recuerda de mis errores y fracasos; no me reconoce por tareas que han requerido mucho esfuerzo.

Escala de liderazgo tóxico (Schmidt, 2008). Medida incluida para obtener evidencias de validez convergente. Schmidt (2008) reporta cuatro dimensiones (excluyendo la supervisión abusiva): Autopromoción ( $\alpha=.91)$; Narcicismo $(\alpha=.88) ; \quad$ Liderazgo autoritario $(\alpha=.89) \quad \mathrm{e}$ Impredecibilidad $(\alpha=.92)$. Popa, et al., (2013) realizaron una adaptación del cuestionario al rumano efectuando un análisis factorial exploratorio y confirmatorio en una muestra de 352 participantes encontrando que las escalas mostraron buenos niveles alfa (.82 a .90$)$ y evidencias de validez convergente y discriminante aceptables. Un hallazgo importante reportado por Popa et al. (2013) es que los ítems de la escala de

Tabla 1. Características de las muestras de estudio

\begin{tabular}{|c|c|c|c|}
\hline & $\begin{array}{c}\text { Muestra } 1 \\
(\mathrm{~N}=308)\end{array}$ & $\begin{array}{c}\text { Muestra } 2 \\
(\mathrm{~N}=283)\end{array}$ & Totales \\
\hline Edad & $M^{I}=31.1 ; D E^{2}=5.6$ & $M=31.5 ; D E=6.6$ & $M=31 ; D E=6$ \\
\hline Mujer & $M=31.6 ; D E=6$ & $M=33.6 ; D E=7.6$ & $M=33 ; D E=7$ \\
\hline Hombre & $M=31.3 ; D E=5.6$ & $M=32.6 ; D E=7.2$ & $M=31.9 ; D E=6.5$ \\
\hline \multicolumn{4}{|l|}{ Sexo } \\
\hline Mujer & $200(33.8)$ & $140(23.7)$ & $340(57.5)$ \\
\hline Hombre & $108(18.3)$ & $143(24.2)$ & $251(42.5)$ \\
\hline \multicolumn{4}{|c|}{ Nivel educativo } \\
\hline Profesional & $99(32.1)$ & $162(57.2)$ & $261(44.2)$ \\
\hline Postgrado & $206(66.9)$ & $109(38.5)$ & $315(53.3)$ \\
\hline Otro & $3(1)$ & $12(4.2)$ & $15(2.5)$ \\
\hline \multicolumn{4}{|c|}{ Tipo de institución } \\
\hline Privada & $198(64.3)$ & $172(60.8)$ & $370(62.6)$ \\
\hline Pública & $102(33.1)$ & $97(34.3)$ & $199(33.7)$ \\
\hline Otra & $8(2.6)$ & $14(4.9)$ & $22(3.7)$ \\
\hline \multicolumn{4}{|l|}{ Edad del jefe ${ }^{3}$} \\
\hline $20-30$ años & $21(6.8)$ & $23(8.1)$ & $44(7.4)$ \\
\hline 31-40 años & $114(37)$ & $134(47.3)$ & $248(42)$ \\
\hline $41-50$ años & $95(30.8)$ & $91(32.2)$ & $186(31.5)$ \\
\hline$>50$ años & $78(25.3)$ & $35(12.4)$ & $113(19.1)$ \\
\hline \multicolumn{4}{|l|}{ Sexo del jefe } \\
\hline Mujer & $140(23.7)$ & $120(20.3)$ & $260(44)$ \\
\hline Hombre & $168(28.4)$ & $163(27.6)$ & $331(56)$ \\
\hline \multicolumn{4}{|c|}{ Nivel educativo del jefe } \\
\hline Profesional & $132(42.9)$ & $144(50.9)$ & $276(46.7)$ \\
\hline Postgrado & $170(55.2)$ & $129(45.6)$ & $299(50.6)$ \\
\hline Otro & $6(1.9)$ & $10(3.5)$ & $16(2.7)$ \\
\hline
\end{tabular}

Nota. $1=$ media; $2=$ desviación estándar. Porcentajes entre paréntesis. $3=$ los participantes proporcionaron los datos de sus jefes directos. 
Tabla 2. Índices de ajuste del Cuestionario de Liderazgo Tóxico

\begin{tabular}{lcc}
\hline & Modelo de dos factores & Modelo de tres factores \\
\hline $\mathrm{X}^{2}$ & 226.837 & 386.250 \\
$\mathrm{~N}$ & 308 & 308 \\
$d f$ & 87 & 147 \\
$\mathrm{TLI}$ & .955 & .937 \\
$\mathrm{CFI}$ & .962 & .946 \\
$\mathrm{RMSEA}$ & .072 & .073 \\
SRMR & .043 & .046 \\
AIC & 292.837 & 472.250 \\
\hline
\end{tabular}

Nota. Dos factores: autopromoción e impredecibilidad. Tres factores: autopromoción, impredecibilidad y liderazgo autoritario.

Tabla 3. Medidas de Validez: Cuestionario de Liderazgo Tóxico

\begin{tabular}{lcccccc}
\hline Factores & $C R$ & $A V E$ & $M S V$ & $\operatorname{Max}(H)^{c}$ & Autopromoción & Impredecibilidad \\
\hline Autopromoción $^{\mathrm{a}}$ & .94 & .64 & .54 & .94 & $\mathbf{8 0}$ & \\
Impredecibilidad $^{\mathrm{b}}$ & .93 & .68 & .54 & .93 & $.73^{* * *}$ & $\mathbf{8 3}$ \\
\hline
\end{tabular}

Nota. $* * * p<.001 . \mathrm{CR}=$ confiabilidad compuesta; AVE=promedio de varianza extraída; MSV=varianza compartida promedio y máxima; $\operatorname{MaxR}(\mathrm{H})=$ confiabilidad máxima. $\mathrm{a}=$ integrada por nueve ítems. $\mathrm{b}=$ compuesta por seis ítems. $\mathrm{c}=\mathrm{confiabilidad}$ máxima (o índice H), considerada una medida más robusta de confiabilidad y que es más apropiada con variables latentes en modelos estructurales. Su umbral mínimo es .80 (Hancock \& Mueller, 2001).

Narcisismo y Autopromoción se condensaron en un solo factor. En el presente estudio al efectuar el análisis exploratorio se encontró que al igual que lo reportado por Popa et al. (2013) los factores de Narcisismo y Autopromoción también se integraron en uno solo, de modo que quedaron tres factores: Autopromoción, Impredecibilidad y Liderazgo autoritario. Sin embargo, como se aprecia en la Tabla 2, al realizar el análisis confirmatorio se optó por la solución de dos factores que presentó los mejores índices de ajuste. Los valores Alfa resultantes fueron: Autopromoción $\quad(\alpha=.94)$ e Impredecibilidad $(\alpha=.93)$.

El factor de Liderazgo autoritario fue descartado ya que si bien tenía un Alfa aceptable $(\alpha=.79)$, mostró problemas de validez discriminante con Autopromoción y de validez convergente pues el promedio de varianza extraída (AVE) fue menor que .50. La Tabla resume las medidas finales de validez del cuestionario.

Las medidas de validez demuestran que ambos factores tienen excelentes niveles de confiabilidad $(C R>.70 ; \quad$ MaxR $>.80)$; validez convergente $(A V E>.50)$; y validez discriminante ya que las raíces cuadradas del $A V E$ (que aparecen en la diagonal al final de la tabla en negritas) son mayores que la correlación entre factores $(r=.73)$.

Por tanto, el cuestionario estuvo finalmente conformado por 15 ítems, agrupados en dos facotres que explicaron el $71 \%$ de la varianza. El cuestionario usa una escala Likert de acuerdo donde $=$ total desacuerdo hasta $5=$ total acuerdo. Ejemplo de ítems: afecta el estado emocional de sus colaboradores cuando se molesta (impredecibilidad), cree que es una persona especial y privilegiada (autopromoción).

Cuestionario de Satisfacción Laboral S20/23 (Meliá \& Peiró, 1989; Chiang, Salazar, \& Núñez, 2007). Cuestionario compuesto por 23 ítems organizados originalmente en cinco factores. Meliá y Peiró (1989) reportaron una estructura factorial de cinco factores que explicaba el $63 \%$ de la varianza total y un índice Alfa de Cronbach (.92) y alfas para cada una de las escalas en el rango .76 a .89. En el presente estudio se efectuó un análisis factorial exploratorio y confirmatorio que resultó en la eliminación de un ítem y la identificación de cinco factores, aunque fue necesario ajustar sus nombres considerando el contenido de los ítems. Los factores son: satisfacción con las características del trabajo, siete ítems, $\alpha=.88$; satisfacción con la participación y la autonomía, seis ítems, $\alpha=.88$; satisfacción con la supervisión, tres ítems, $\alpha=.91$; satisfacción con las condiciones físicas, cuatro ítems, $\alpha=.83$ y satisfacción con el cumplimiento de normas, dos ítems, $\alpha=.82$. Se responde en una escala Likert de cinco puntos cuyos extremos son $1=$ total desacuerdo hasta $5=$ total acuerdo. Ejemplos: ¿qué tan satisfecho está con la 
oportunidad que le ofrece su trabajo de hacer las cosas que le gustan (características del trabajo); la capacidad para decidir autónomamente aspectos de su trabajo (participación y autonomía); las relaciones personales con su jefe (supervisión); la ventilación (condiciones físicas); el grado en que su institución cumple con normas y leyes de trabajo (cumplimiento de normas).

Cuestionario LMX-7 (Nader \& Castro, 2010). Basado en la Teoría del intercambio lídermiembro (LMX) (Gerstner \& Day, 1997). Es una medida de liderazgo que mide la calidad de la relación líder - colaborador. Se la incluye para buscar evidencias de validez discriminante. Se usa la versión corta, de siete ítems, donde cada una de las preguntas se contesta con un formato Likert de cinco opciones. Ejemplos de ítems: ¿conoce qué opinión tiene su jefe del trabajo que usted realiza?; ¿tengo la suficiente confianza en mi jefe como para defender y justificar sus decisiones si él/ella no estuviera presente para hacerlo? Nader $\&$ Castro (2010) reportaron un Alfa de .75. En la muestra actual el cuestionario reportó un Alfa de $\alpha=.93$.

\section{Medidas aplicadas en la segunda muestra}

Aparte de la Escala de supervisión abusiva se emplearon las siguientes medidas.

Escala de intención de rotación, TIS-6 (Bothma \& Roodt, 2013). Tiene seis ítems que se contestan con una escala Likert de cinco puntos cuyas descripciones son específicas para cada pregunta. Ejemplo de ítems: ¿Con qué frecuencia ha considerado la opción de conseguir otro trabajo que se ajuste más a sus necesidades e intereses?, ¿Qué tan probable es que usted acepte un trabajo diferente por la misma paga? Bothma y Roodt (2013), reportaron una estructura de un solo factor, un Alfa de $.80 \mathrm{y}$ capacidad predictiva de la rotación de personal como tal. La escala presentó en la muestra de estudio las siguientes propiedades: un solo factor que explica el $69 \%$ de la varianza, con cargas factoriales desde .54 a .88 , un coeficiente Alfa $(\alpha=.90)$ y los siguientes índices: $\chi^{2} \quad(8)=43.24, \quad p>.001 ; \quad R M S E A=.10$; $S R M R=.049 ; C F I=.98$ y $T L I=.97$, que demuestran un muy buen ajuste.

Maslach Burnout Inventory General Survey, MBI - GS (Juárez, García, Camacho, Gómez, Vera, Fernández, \& García, 2011;
Fernández, Juárez, \& Merino, 2015). Se usó la versión en español, traducida para América Latina (Juárez et al., 2011) compuesta por 16 ítems que miden tres factores: Agotamiento emocional (cinco ítems), Eficacia profesional (seis ítems) e Indiferencia (cinco ítems). Utiliza una escala Likert de siete puntos donde $0=$ nunca y $6=$ todos los días. Ejemplo de los ítems: me siento agotado emocionalmente por mi trabajo (agotamiento); puedo resolver eficazmente los problemas que surgen en mi trabajo (eficacia); ha disminuido mi interés por el trabajo desde que comencé en este empleo (indiferencia). El análisis factorial exploratorio corroboró la estructura de tres factores que explicaron un 63\% de la varianza con los siguientes coeficientes Alfa: Agotamiento $(\alpha=.88)$, Eficacia $(\alpha=.82)$ e Indiferencia $(\alpha=.82)$. $\mathrm{El}$ análisis confirmatorio resultó en estos índices: $\chi 2(72)=197.55, p<.001 ; R M S E A=.06 ; S R M R=.06$; $C F I=.96$ y $T L I=95$, evidenciando un buen ajuste. Las medidas de validez convergente $y$ discriminante entre las escalas también resultaron apropiadas.

Escala Utrech de "Engagement" en el trabajo, UWES-17 (Schaufeli \& Bakker, 2003; Hernández, Llorens, Rodríguez, \& Dickinson, 2016). La escala tiene 17 ítems que miden tres factores: vigor, dedicación y absorción. Las preguntas se califican con una escala Likert de 7 puntos donde $0=$ ninguna vez hasta $6=$ todos los días. El resultado del análisis factorial exploratorio y confirmatorio, en la presente muestra, sugiere que, a diferencia de lo reportado en el manual original y lo encontrado en un estudio de adaptación de la escala al español (Hernández et al., 2016), la estructura está definida por un solo factor que explica el 63\% de la varianza y está integrada por 12 ítems que muestran un coeficiente Alfa ( $\alpha=.95)$. Este factor, por su contenido, se refiere al "vigor" que es el componente principal del compromiso laboral (Shirom, 2010). Ejemplos de ítems: estoy entusiasmado con mi trabajo, me siento fuerte y vigoroso en mi trabajo.

\section{Procedimiento}

La traducción de la Escala de SA se realizó en varios pasos conforme lo sugerido por Guillemin, Bombardier y Beaton (1993) y considerando las guías de la "International Test Commission" 
(Hambleton, 2001). La escala pasó por las etapas de traducción, retro-traducción, evaluación de la traducción inicial, revisión de las versiones y prueba piloto con un grupo de 22 personas para que hicieran observaciones respecto a la claridad de las instrucciones y los ítems.

Se tomó la decisión de aplicar la escala a dos muestras separadas por un intervalo de seis meses. Esto se hizo considerando que no se podían aplicar todas las medidas a la vez, dado el limitado tiempo que se otorgó a los investigadores para trabajar con los participantes. Además, el tener dos muestras separadas en el tiempo permite aplicar una prueba de invarianza factorial que también ofrece evidencias de validez de constructo (Barrera-Barrera, Navarro-García, \& Peris-Ortiz, 2015).

La escala se aplicó a ambas muestras de manera presencial, entregando a los participantes un consentimiento informado que fue leído en voz alta, explicándoles el propósito del estudio, asegurando la confidencialidad y respetando el carácter voluntario de la participación.

La información fue codificada y transcrita. Se hicieron varias revisiones para eliminar cualquier error de transcripción y asegurar que todos los valores de las diversas variables estuvieran dentro de los rangos posibles. Al final se encontró que había un bajo porcentaje de casos perdidos inferior al $1.4 \%$ en algunas variables. Se procedió entonces a reemplazar estos valores con la mediana de puntos adyacentes para las variables ordinales y la media de puntos adyacentes para las variables continuas (Little \& Rubin, 2014). También se eliminaron unos pocos casos que tenían varianza cero en algunas variables o un elevado número de omisiones.

Dado que la escala fue aplicada a dos muestras con un espacio de seis meses, se efectuó una prueba de invarianza configuracional con un modelo libre en los dos grupos; una prueba de invarianza métrica mediante la restricción de los dos modelos para forzar su igualdad y una prueba de diferencia de Chi cuadrado entre los modelos libre y restringido para verificar la invarianza de los parámetros entre los grupos. Comprobada la invarianza de los grupos, el análisis factorial exploratorio y confirmatorio de la escala de SA se hizo con la muestra total $(\mathrm{N}=591)$. En el análisis factorial exploratorio se utilizó el método de extracción la Factorización de ejes principales. Para tener mayor seguridad en el número de factores se usó el análisis paralelo de Horn (Horn, 1965) con el sistema RanEigen (Enzmann, 1997) que genera autovalores aleatorios considerando el tamaño de la muestra y el número de ítems de la escala.

Tepper (2007) reporta que la SA es un fenómeno de un bajo índice de frecuencia base, lo que en el presente estudio se reflejó en valores extremos de asimetría y curtosis en la mayoría de ítems de la escala de SA, que llevaron a descartar el supuesto de distribución normal multivariada a nivel poblacional, de modo que fue necesario usar, para el análisis factorial confirmatorio, el software R versión 3.4 .2 (2017) con el paquete "Lavaan" (latent variable analysis) (Rosseel, 2012) que ofrece el estimador WLSMV (weighted least squares with mean and variance adjustment). Según Brown (2006): "si uno o más de los indicadores de los factores es categórico (o la no-normalidad es extrema), la teoría normal (máxima verosimilitud) no debería usarse (...); el WLSMV es un estimador robusto que no asume variables distribuidas normalmente y proporciona la mejor opción para el modelado de datos categóricos u ordinales" (p. 76). Los índices de ajuste que se analizaron fueron: chi-cuadrado $\left(\chi^{2}\right)$ donde el modelo tiene un ajuste adecuado para valores $\chi 2$ / gl entre 1 y 3 (Carmo, Brás \& Batista, 2017; Kline, 2011); el índice de ajuste comparativo (CFI) y el índice Tucker-Lewis (TLI) donde valores iguales o mayores a .90 son deseables (Bentler, 1990); el error cuadrático medio de aproximación (RMSEA) que debe tener un valor igual o menor a .06 para considerar un excelente ajuste del modelo y la raíz media cuadrática residual estandarizada (SRMR) donde lo ideal es un valor menor a $.08(\mathrm{Hu} \&$ Bentler, 1999).

Como el supuesto de normalidad fue descartado, las correlaciones que se calcularon para obtener las evidencias de validez convergente y discriminante se hicieron con el uso de simulador de muestras (bootstrap) con 5000 iteraciones, intervalo de confianza del $95 \%$ y corrección por sesgo (bias-corrected and accelerated), de modo que se reportará además del valor $p$ tradicional, los intervalos de confianza de cada correlación. 
Para el análisis de confiabilidad y para mantener la coherencia con el estimador empleado en el análisis confirmatorio de la Escala de SA, se usó el Alfa ordinal que ofrece una estimación más exacta para respuestas tipo Likert y datos no normales (Gadermann, Guhn, \& Zumbo, 2012). En las demás medidas se usó el Alfa de Cronbach (Cronbach, 1951).

Se obtuvieron los estadísticos descriptivos y se verificó si la media de la escala se encontraba en rango reportado en la literatura.

\section{Resultados}

\section{Evidencia de validez de contenido}

Después de traducir la escala y de realizar el piloto con 22 personas, se pidió a un grupo de 14 psicólogos que estimaran la validez de contenido de la escala calificando cada uno de los ítems con los criterios propuestos por Cassepp-Borges, Balbinotti y Teodoro (2010), esto es: claridad del lenguaje; pertinencia práctica y relevancia teórica. La calificación independiente de los 14 jueces dio un coeficiente corregido de validez de contenido de .97 para la Escala de supervisión abusiva.

\section{Evidencia respecto a la estructura factorial de la Escala de supervisión abusiva}

Inicialmente se hizo el análisis factorial exploratorio (AFE) y confirmatorio (AFC) con la primera muestra $(\mathrm{N}=308)$; sin embargo, una vez que se verificó la invarianza de los grupos, se reportan a continuación los resultados para la muestra total $(\mathrm{N}=591)$.

El estadístico Kaiser-Meyer-Olkin (KMO) fue de .94 con un valor significativo para el Test de esfericidad de Bartlett $\chi 2(105)=4521.9, p>.001$ lo que evidencia adecuación muestral.

El (AFE) se hizo con factorización de ejes principales y extracción con el criterio GuttmanKaiser (Guttman, 1954; Kaiser, 1960) que sugirió inicialmente dos factores. Sin embargo, tomando en cuenta las recomendaciones de Buja y Eyboglu (1992) y Zwick y Velicer (1986) sobre el número de factores a extraer, se usó el análisis paralelo de Horn, con el software RanEigen (Enzmann, 1997), resultando en la identificación de un solo factor que explicaba el $49.44 \%$ de la varianza. Las cargas factoriales de los ítems tuvieron valores desde .54 hasta .79 (Tabla 4).
Tabla 4. Cargas Factoriales de la Escala de Supervisión Abusiva

\begin{tabular}{|c|c|c|}
\hline No. & Ítems & Cargas \\
\hline 4 & Me critica en frente de otras personas & .79 \\
\hline 8 & $\begin{array}{l}\text { Me echa la culpa para quedar bien } \\
\text { ante otros }\end{array}$ & .77 \\
\hline 11 & $\begin{array}{l}\text { Hace comentarios negativos de mi } \\
\text { persona a los demás }\end{array}$ & .76 \\
\hline 12 & Es grosero conmigo & .74 \\
\hline 1 & $\begin{array}{l}\text { Me hace quedar en ridículo ante los } \\
\text { demás }\end{array}$ & .73 \\
\hline 10 & $\begin{array}{l}\text { Se enfada conmigo por motivos } \\
\text { ajenos a mi persona }\end{array}$ & .69 \\
\hline 15 & Me miente & .68 \\
\hline 6 & $\begin{array}{l}\text { Me recuerda de mis errores y fracasos } \\
\text { pasados }\end{array}$ & .68 \\
\hline 2 & Me dice que mis ideas son absurdas & .67 \\
\hline 7 & $\begin{array}{l}\text { No me da el crédito por tareas que } \\
\text { han requerido mucho esfuerzo de mi } \\
\text { parte }\end{array}$ & .64 \\
\hline 3 & Me aplica la "ley del hielo" & .62 \\
\hline 5 & Invade mi privacidad & .62 \\
\hline 9 & No cumple las promesas ofrecidas & .60 \\
\hline 13 & $\begin{array}{l}\text { No me permite interactuar con mis } \\
\text { colegas }\end{array}$ & .59 \\
\hline 14 & Me dice que soy incompetente & .54 \\
\hline
\end{tabular}

Tabla 5. Índices de Ajuste de la Escala de Supervisión Abusiva

\begin{tabular}{lc}
\hline Índices & Valores \\
\hline $\mathrm{X}^{2}$ & 312.88 \\
$\mathrm{~N}$ & 591 \\
$d f$ & 90 \\
$\mathrm{CFI}$ & .97 \\
$\mathrm{TLI}$ & .97 \\
$\mathrm{RMSEA}$ & .06 \\
$\mathrm{SRMR}$ & .04 \\
Nota. Se usó el estimador WLSMV (weighted least \\
squares with mean and variance adjustment).
\end{tabular}

El AFC se lo hizo con el software R, paquete Lavaan (Rosseel, 2012). Los índices obtenidos demuestran un excelente ajuste del modelo (Tabla $5)$.

\section{Confiabilidad de la escala}

Respecto a la confiabilidad de la Escala se encontró un Alfa ordinal =.95 (siendo este coeficiente el más apropiado cuando no se cumple el supuesto de normalidad). De todas formas, puesto que en la literatura sobre la SA se reporta la mayoría de las veces el Alfa de Cronbach, el valor fue: $\alpha=.92$.

\section{Evidencia respecto a la invarianza de medición entre grupos}

Dado que la escala se aplicó a dos grupos con 
Tabla 6. Supervisión Abusiva: Evidencias de Validez Convergente y Discriminante

\begin{tabular}{|c|c|c|c|c|}
\hline Variables & Correlaciones & Error estándar & Límite inferior & Límite superior \\
\hline \multicolumn{5}{|l|}{ Validez convergente } \\
\hline Autopromoción ${ }^{1}$ & $.72 * *$ & .027 & .664 & .773 \\
\hline Impredecibilidad $^{1}$ & $.64 * *$ & .037 & .563 & .712 \\
\hline Agotamiento emocional $^{2}$ & $.27 * *$ & .063 & .143 & .392 \\
\hline Indiferencia ${ }^{2}$ & $.24 * *$ & .066 & .106 & .371 \\
\hline Intención de rotación ${ }^{2}$ & $.32 * *$ & .067 & .178 & .45 \\
\hline \multicolumn{5}{|l|}{ Validez discriminante } \\
\hline $\begin{array}{l}\text { Satisfacción - características } \\
\text { del trabajo }\end{array}$ & $-.43 * *$ & .051 & -.52 & -.32 \\
\hline $\begin{array}{l}\text { Satisfacción - participación y } \\
\text { autonomía }^{1}\end{array}$ & $-.48 * *$ & .044 & -.557 & -.387 \\
\hline Satisfacción - supervisión ${ }^{1}$ & $-.71 * *$ & .032 & -.768 & -.651 \\
\hline $\begin{array}{l}\text { Satisfacción - condiciones } \\
\text { físicas }{ }^{1}\end{array}$ & $-.14 * *$ & .061 & -.255 & -.007 \\
\hline $\begin{array}{l}\text { Satisfacción - complimiento } \\
\text { de normas }{ }^{1}\end{array}$ & $-.19 * *$ & .054 & -.295 & -.078 \\
\hline Satisfacción laboral general $^{1}$ & $-.53 * *$ & .040 & -.601 & -.448 \\
\hline LMX- $7^{1}$ & $-.64 * *$ & .031 & -.696 & -.582 \\
\hline Autoeficacia profesional $^{2}$ & $-.17 * *$ & .064 & -.297 & -.048 \\
\hline Vigor $^{2}$ & $-.24 * *$ & .076 & -.39 & -.086 \\
\hline
\end{tabular}

un intervalo de seis meses, se efectuó primero una prueba de invarianza configuracional o de estructura, obteniéndose una bondad de ajuste adecuada cuando se analizó un modelo de estimación libre entre los grupos. Luego se efectuó una prueba de invarianza métrica mediante la restricción de los grupos para que sean iguales y se hizo una prueba de diferencias de Chi cuadrado entre el modelo restringido y el no restringido, encontrándose que eran invariantes, $p=.29$.

\section{Evidencias de validez convergente y discriminante}

En la primera muestra $(\mathrm{N}=308)$ la Escala de SA se correlacionó con las siguientes escalas: Autopromoción, Impredecibilidad, Satisfacción con las características del trabajo, Satisfacción con la participación y autonomía, Satisfacción con la supervisión, Satisfacción con las condiciones físicas, Satisfacción con el cumplimiento de normas, Satisfacción laboral general y Calidad de la relación jefe - colaborador (LMX).

En la segunda muestra $(\mathrm{N}=283)$ la escala de SA se correlacionó con: Agotamiento emocional, Indiferencia, Intención de rotación y Vigor (Tabla $6)$.

\section{Resultados descriptivos}

$\mathrm{Se}$ encontraron las siguientes medias aritméticas de la Escala de SA en las muestras: grupo $1 \quad(N=308, M=1.49, D S=.63)$; grupo 2 $(N=283, M=1.33, D S=.52)$ y grupo total $(N=591$, $M=1.48, D S=.63$ ) que están dentro de los rangos de las medias aritméticas encontradas en estudios previos; 1.38 (Tepper, 2000); 1.26 (Tepper, Duffy, Hoobler, \& Ensley, 2004), y 1.87 (Aryee, Sun, Chen, \& Debrah, 2008).

\section{Discusión}

El objetivo del presente artículo fue traducir, adaptar e investigar las evidencias de validez de la Escala de supervisión abusiva (Tepper, 2000). Para esto se efectuaron procedimientos de traducción y se aplicaron varios instrumentos, en dos muestras, para evaluar tanto la estructura factorial de la escala como las evidencias de validez convergente y discriminante.

El análisis factorial exploratorio demostró que los 15 ítems de la escala saturaban en un solo factor latente que explicaba el $49 \%$ de la varianza total con cargas factoriales desde .54 a .79 . El análisis factorial confirmatorio reveló igualmente que la mejor estructura factorial se conseguía con el modelo de un solo factor latente como lo sugiere el conjunto de índices propios de este análisis. La prueba de invarianza factorial también aportó evidencias de validez de constructo pues se encontró que la estructura factorial resultó 
invariante entre ambos grupos en los que la escala se aplicó con un intervalo de seis meses.

Al examinar los tres primeros ítems que tienen las cargas factoriales más altas (Tabla 4), se observa que tienen en común la descripción de acciones hostiles que el supervisor le hace al colaborador en frente de otras personas (me critica en frente de otros; me echa la culpa para quedar bien y hace comentarios negativos de mi persona a los demás). Es decir, el escarnio público de los colaboradores es lo que esencialmente define al jefe abusivo.

Respecto a las relaciones significativas encontradas se constata que todas ellas son consistentes con lo reportado en la literatura. Así, la SA se asocia principalmente con otros estilos negativos de liderazgo como la impredecibilidad (los exabruptos que sorprenden al colaborador) y la auto promoción (que contiene elementos de narcisismo). La evidencia de validez convergente también sugiere que la SA se relaciona con el agotamiento emocional (el núcleo del síndrome de quemado laboral, Pedrosa \& García-Cueto, 2016), la indiferencia y la intención de rotación, hallazgos que en su conjunto sugieren que la SA tiene consecuencias serias en el bienestar psicológico de los trabajadores.

La SA también afecta negativamente la satisfacción laboral general, de manera especial la satisfacción con la supervisión y la satisfacción con la participación y la autonomía, coincidiendo con lo reportado en algunos estudios (Hsu \& Wu, 2016; Mackay et. al. (2015). Perilla-Toro y Gómez-Ortiz (2017) afirman que la satisfacción laboral es una forma de bienestar afectivo, de modo que la correlación hallada también constituye evidencia de que la SA se asocia negativamente con el bienestar afectivo de los colaboradores.

La SA se relaciona negativamente con la calidad de la relación líder-colaborador (LMX), el vigor (principal componente del compromiso laboral) y la autoeficacia profesional. El menoscabo de las relaciones jefe-colaborador y la insatisfacción con la supervisión crea las condiciones para intercambios hostiles y el uso de tácticas negativas de influencia social como el control psicológico por parte del jefe directo (Tepper \& Almeda, 2012). No se encontraron relaciones significativas entre la SA y las variables demográficas del supervisado y el supervisor. El meta análisis de Mackay et. al. (2015) reporta un hallazgo similar que, según los mencionados autores, significa que una "amplia gama de empleados reporta las percepciones de supervisión abusiva” (p. 1954).

Un hallazgo interesante es que la media aritmética de la Escala de SA en la muestra total $(\mathrm{N}=591, \mathrm{M}=1.48)$ es similar a la encontrada en estudios previos (Aryee et al., 2008; Tepper, 2000; Tepper, Duffy, Hoobler y Ensley (2004). El meta análisis de Mackey et. al., (2015) reporta las medias y otros descriptivos que se han obtenido de la escala en 11 países diferentes encontrándose que los descriptivos del presente estudio se acercan más a los encontrados en países del hemisferio occidental (Bélgica, $\mu=1.47$; Canadá, $\mu=1.53$; Estados Unidos, $\mu=1.68$ ) que a los encontrados en países del hemisferio oriental (China, $\mu=2.06$; Filipinas, $\mu=2.17$; Taiwán, $\mu=2.13$ ), aunque también hay excepciones (India, $\mu=1.67)$. Este resultado lleva a considerar el papel que juega la cultura en la percepción de abuso por parte de los colaboradores.

En suma, el conjunto de evidencias analizadas en este estudio sugiere que la escala de SA tiene validez de constructo. Sin embargo, la validez no se puede establecer con un solo estudio, de modo que se necesitarán a futuro nuevas investigaciones que permitan evaluar a la escala (Shadish, Cook \& Campbell, 2001).

\section{Sugerencias para futuras investigaciones}

Siendo este el primer estudio que realiza la traducción y adaptación de la escala de Tepper (2000) al castellano, se avizoran algunas líneas de investigación: hace falta explorar la relación de la SA con otras variables criterio como las conductas cívicas organizacionales, las conductas contraproducentes y otros indicadores de bienestar psicológico en el trabajo.

Indagar las variables mediadoras entre la SA y ciertas variables criterio, en especial sería importante modelar el papel de las percepciones de justicia organizacional (Greenberg \& Colquitt, 2013) ya que esta teoría fue el primer marco conceptual que usó Tepper (2000) para analizar la mediación entre la SA y algunas variables criterio. Otra teoría que tiene el potencial de explicar los mecanismos mediadores que operan en la 
percepción de la SA es la Teoría de la Autodeterminación (TAD) (Ryan \& Deci, 2017). Un estudio de Lian, Ferris y Brown (2012a) comparó los constructos de la TAD con la Teoría de la justicia organizacional y la Teoría del intercambio social como mediadores de la relación entre SA y conductas contraproducentes, encontrando que la TAD explicaba una mayor cantidad de varianza. Sería relevante analizar, desde los planteamientos de esta teoría, cómo un estilo abusivo frustra la satisfacción de las necesidades psicológicas básicas induciendo una motivación controlada que finalmente se asocia a consecuencias negativas como la intención de rotación y el síndrome de quemado laboral.

La cultura juega un papel importante en las percepciones de justicia y consecuentemente en las actitudes y los comportamientos de la gente (Rupp, 2011), de modo que se hace necesario evaluar cómo las percepciones de SA están moderadas por variables culturales.

Los antecedentes de la SA han sido menos investigados que las consecuencias (Zhang \& Bednall, 2015), mas es claro que se trata de una línea de investigación por desarrollar, en especial los diversos tipos de antecedentes que se han planteado (antecedentes relacionados con el supervisor, con el supervisado y con las características de la organización).

La SA se ha estudiado principalmente como un fenómeno de nivel individual o micro. Sin embargo, hay evidencia de que también se manifiesta a nivel meso en el clima de los equipos de trabajo (Priesemuth, et al., 2014). Como afirman estos últimos autores, la $\mathrm{SA}$ no es un problema cuya afectación se limita a unos pocos individuos aislados, sino que también causa estragos a grupos y organizaciones enteras.

La SA también puede ser estudiada en el contexto de las prácticas de gestión del talento que se enfocan en mejorar el desempeño y la productividad de personas y grupos mediante los llamados "sistemas laborales de alto rendimiento" (Ashkanasy, Bennett, \& Martinko, 2016). Jensen y Van De Voorde (2016) sugieren que estas prácticas pueden tener un lado oscuro que vuelve tenue la línea entre motivación y abuso, de modo que es necesario estudiar las circunstancias bajo las cuales una práctica de talento, originalmente destinada a mejorar la motivación y el desempeño, desemboca en supervisión abusiva y en un clima de temor y agresión interpersonal generalizada.

Así, el establecimiento de estrategias que permitan la prevención, el tratamiento y el control de este riesgo psicosocial en las organizaciones (Fernades \& Pereira, 2016), debe sustentarse en hallazgos empíricos y desarrollos teóricos que orienten en el diseño y la implementación de intervenciones efectivas.

\section{Recomendaciones de orden metodológico}

Acogiendo la recomendación de Mackey et al., (2015) se sugiere la aplicación completa de la escala de SA, y no de formas abreviadas, en los diversos estudios que se efectúen, para facilitar las comparaciones y el análisis de invarianza multigrupos.

En el presente estudio se constató que el supuesto de normalidad multivariada no se cumplía con los datos recolectados; la SA es un fenómeno de bajo índice de frecuencia base (Tepper, 2007). Se recomienda que los investigadores verifiquen esta condición pues si se presenta se deberían escoger procedimientos y estimadores apropiados.

\section{Limitaciones}

Los cuestionarios fueron llenados mediante auto reporte, lo que conlleva el sesgo de varianza por método común. Una limitante fue no haber incluido medidas para controlar y remover esta varianza como han recomendado Podsakoff, MacKenzie, Lee, \& Podsakoff (2003). De todas formas, los resultados descriptivos y las propiedades psicométricas de la escala son consistentes con lo reportado en la literatura lo que lleva a concluir que la influencia del sesgo no ha llegado a un nivel que se pueda considerar problemático. Es recomendable que en futuros estudios se incorporen medidas para aislar la varianza por método común. Harvey, Butler y Brees (2016) desarrollaron una medida específica, basada en rasgos afectivos, para remover la varianza de método común en la escala de SA.

Otra limitación tuvo que ver con el perfil demográfico de las muestras que estuvieron compuestas, en general, por personas jóvenes $\left(M_{\text {edad }}=31\right.$ años $)$, con buen nivel educativo $\mathrm{y}$ trabajando mayoritariamente en empresas privadas. Esto limita la validez externa de los 
resultados. Es recomendable que a futuro se realicen estudios con muestras cuyas características demográficas sean diferentes.

Se usaron dos medidas que no han sido previamente traducidas al castellano: el Cuestionario de liderazgo tóxico (Schmidt, 2008) y la Escala de intención de rotación (Bothma \& Roodt, 2013). La primera medida se escogió porque hasta donde se pudo indagar, no se encontró una escala en castellano que midiera estilos negativos de liderazgo. En la estrategia de validación se planteó la necesidad de relacionar la escala de SA con otros estilos negativos de liderazgo como evidencia de validez convergente. Respecto a la segunda medida tampoco se pudo encontrar en castellano una escala breve y confiable que midiera el constructo de interés. Lo que se hizo fue aplicar los mismos procedimientos de traducción y adaptación que se aplicaron a la escala de SA a estas medidas. También se hizo el análisis factorial exploratorio y confirmatorio, y se verificó la validez convergente y discriminante. Aparte de que las propiedades psicométricas de ambas medidas resultaron similares a estudios previos (Popa, et al., 2013; Bothma \& Roodt, 2013), los patrones de convergencia y discriminancia fueron los esperados y los índices de ajuste de ambas medidas fueron muy buenos.

Las investigaciones en Iberoamérica sobre la incidencia y las consecuencias de la supervisión abusiva son incipientes. Si bien hay alguna literatura sobre el acoso laboral (RodríguezMuñoz, 2011; Topa, Depolo, \& Morales, 2007), la investigación sistemática de la SA en países de habla castellana y portuguesa es una asignatura pendiente. Así, el presente estudio busca establecer un punto de partida mediante el establecimiento de la escala base para medir el constructo de interés de forma confiable y válida. Comprender la prevalencia y las consecuencias de la SA en las organizaciones es indispensable para el desarrollo de estrategias de intervención que afronten de manera efectiva un fenómeno que amenaza seriamente el bienestar psicológico de los trabajadores y la productividad.

\section{Referencias}

Ambrose, M. L., Schminke, M., \& Mayer, D. M. (2013). Trickle-down effects of supervisor perceptions of interactional justice: A moderated mediation approach. Journal of Applied Psychology, 98(4), 678. doi:10.1037/a0032080

Aryee, S., Chen, Z. X., Sun, L. Y., \& Debrah, Y. A. (2007). Antecedents and outcomes of abusive supervision: Test of a trickle-down model. Journal of Applied Psychology, 92(1), 191. doi:10.1037/0021-9010.92.1.191

Aryee, S. Sun, L. Chen, Z., \& Debrah, A. (2008). Abusive supervision and contextual performance: The mediating role of emotional exhaustion and the moderating role of work unit structure. Management and Organization Review, 4, 393-411. doi:10.1111/j.1740-8784.2008.00118.x

Ashforth, B. (1994). Petty tyranny in organizations. Human Relations, 47, 755-778.

Ashkanasy, N. M., Bennett, R. J., \& Martinko, M. J. (Eds.). (2016). Understanding the highperformance workplace: The line between motivation and abuse. New York: Routledge.

Bamberger, P. A., \& Bacharach, S. B. (2006). Abusive supervision and subordinate problem drinking: Taking resistance, stress and subordinate personality into account. Human Relations, 59(6),723-752. doi:10.1177/0018726706066852

Barrera-Barrera, R., Navarro-García, A., \& PerisOrtiz, M. (2015). El papel de la invarianza factorial en la validación del constructo calidad de servicio electrónico. Revista Europea de Dirección y Economía de la Empresa, 24(1), 13-24. doi.org/ 10.1016/ j.redee.2014.07.001

Bentler, P. M. (1990). Comparative fit indexes in structural models. Psychological Bulletin, 107(2), 238. doi:10.1037/0033-2909.107.2.238

Boddy, C. (2011). Corporate psychopaths, bullying and unfair supervision in the workplace. Journal of Business Ethics, 100(3), 367-379. doi:10.1007/s10551-010-0689-5

Bothma, C. F., \& Roodt, G. (2013). The validation of the turnover intention scale. $S A$ Journal of Human Resource Management, 11(1), 1-12. doi:10.4102/sajhrm.v11i1.507

Brown, T. A. (2006). Confirmatory factor analysis for applied research. New York: The Guilford Press. 
Buja, A., \& Eyuboglu, N. (1992). Remarks on parallel analysis. Multivariate Behavioral Research, 27, 509-540. doi:10.1207/ s15327906mbr2704_2

Burns, W. A. (2017). A descriptive literature review of harmful leadership styles: Definitions, commonalities, measurements, negative impacts, and ways to improve these harmful leadership styles. Creighton Journal of Interdisciplinary Leadership, 3(1), 33-52. doi:10.17062/cjil.v3i1.53

Carlson, D., Ferguson, M., Hunter, E., \& Whitten, D. (2012). Abusive supervision and workfamily conflict: The path through emotional labor and burnout. The Leadership Quarterly, 23(5), 849-859.

doi:10.1016/j.leaqua.2012.05.003

Carmo, C., Brás, M., Batista, L., \& Faísca, L. (2017). Confirmatory factor analysis of the Portuguese version of the Frost multidimensional perfectionism scale. Revista Iberoamericana de Diagnóstico y Evaluación - e Avaliação Psicológica, 44(2), 28-43. doi:10.21865/RIDEP44.2.03

Cassepp-Borges, V., Balbinotti, M. A., \& Teodoro, M. L. (2010). Tradução e validação de conteúdo: Uma proposta para adaptação de instrumentos. Em Pasquali, Luiz. (Ed.), Instrumentação psicológica: fundamentos $e$ práticas (pp. 506-520). Porto Alegre: Artmed Editora.

Chiang, M., Salazar, C.M., Núñez, A. (2007). Clima organizacional y satisfacción laboral en un establecimiento de salud estatal: Hospital tipo 1. Theoria, 16(2), 61-76. Disponible en: www.redalyc.org/articulo.oa?id=29916206

Cronbach, L. J. (1951). Coefficient alpha and the internal structure of tests. Psychometrika, 16(3), 297-334. doi:10.1007/BF02310555

Enzmann, D. (1997). RanEigen: A program to determine the parallel analysis criterion for the number of principal components. Applied Psychological Measurement, 21, 232. doi:10.1177/01466216970213003

Fernández-Arata, M., Juárez, A., \& Merino, C. (2015). Análisis estructural e invarianza de medición del MBI-GS en trabajadores peruanos. Liberabit Revista de Psicología, 21(1), 9-20. Disponible en: http://www.redalyc.org/articulo.oa?id=686395 80002
Fernandes, C., \& Pereira, A. (2016). Exposure to psychosocial risk factors in the context of work: A systematic review. Revista de Saúde Pública, 50. doi:10.1590/S1518-8787.2016050006129

Gabler, C. B., \& Hill, R. P. (2015). Abusive supervision, distributive justice, and work-life balance: Perspectives from salespeople and managers. Journal of Personal Selling \& Sales Management, 35(3), 247-261. doi:10.1080/08853134.2015.1058167

Gadermann, A. M., Guhn, M., \& Zumbo, B. D. (2012). Estimating ordinal reliability for Likert-type and ordinal item response data: A conceptual, empirical, and practical guide. Practical Assessment, Research \& Evaluation, 17(3). Disponible en: www.pareonline.net/getvn.asp? $\mathrm{v}=17 \& \mathrm{n}=3$

Gerstner, C. R., \& Day, D. V. (1997). Metaanalytic review of leader-member exchange theory: Correlates and construct issues. Journal of Applied Psychology, 6(82), 827844. doi:10.1037/0021-9010.82.6.827

Greenberg, J., \& Colquitt, J. A. (Eds.). (2013). Handbook of organizational justice. Psychology Press.

Guillemin, F., Bombardier, C. \& Beaton D. (1993) Cross-cultural adaptation of health-related quality of life measures: Literature review and proposed guidelines. Journal of Clinical Epidemiology, 46, 1417-1432. doi:10.1016/0895-4356(93)90142-N

Guttman, L. (1954). Some necessary conditions for common factor analysis. Psychometrika, 19, 149-162. doi:10.1007/BF02289162

Hambleton, R. K. (2001). The next generation of the ITC test translation and adaptation guidelines. European Journal of Psychological Assessment, 17(3), 164-172. doi:10.1027//1015-5759.17.3.164

Hancock, G. R., \& Mueller, R. O. (2001). Rethinking construct reliability within latent variable systems. Structural Equation Modeling: Present and future, 195-216.

Harvey, P., Butler, F., \& Brees, J. (2016). Propensity to perceive abusive supervision. In Ashkanasy, N. M., Bennett, R. J., \& Martinko, M. J. (Eds.), Understanding the highperformance workplace: The line between motivation and abuse (pp. 191-204). New 
York:Routledge.

doi:10.5465/AMBPP.2015.12482abstract

Hernández Vargas, C. I., Llorens Gumbau, S., Rodríguez Sánchez, A. M., \& Dickinson Bannack, M. E. (2016). Validación de la escala UWES-9 en profesionales de la salud en México. Pensamiento Psicológico, 14(2), 89-100.

Hernández Palomino, J., Espinoza, J. D. J., \& Aguilar Arellano, M. (2013). Clima Laboral: La influencia de la supervisión, los factores organizacionales y la predisposición de los empleados. Revista Internacional Administración \& Finanzas, v. 6(6) pp. 55-71. Disponible en: https://papers.ssrn.com/sol3/ papers.cfm?abstract_id=2285760

Hoobler, J., \& Brass, D. (2006). Abusive supervision and family undermining as displaced aggression. Journal of Applied Psychology, 91, 1125-1133. doi:10.1037/0021-9010.91.5.1125

Horn, J. L. (1965). A rationale and test for the number of factors in factor analysis. Psychometrika, 30(2), 179-185.

Hsu, T. S., \& Wu, C. T. (2016). A Study on the relationship between abusive supervision and job satisfaction: The mediating and moderating effects of organizational politics and organizational justice. Jiaoyu $Y u$ Xinli Yanjiu, 39(4), 1. doi:10.1016/j.rpto.2017.05.002

Hu, L. T., \& Bentler, P. M. (1999). Cutoff criteria for fit indexes in covariance structure analysis: Conventional criteria versus new alternatives. Structural equation modeling: a multidisciplinary journal, 6(1), 1-55. doi:10.1080/10705519909540118

Indradevi, R. (2016). Toxic leadership over the years: A review. Purushartha: A Journal of Management Ethics and Spirituality, 9(1).

Jian, Z., Kwong, H., Qiu, Q., Qiang, Z., \& Hongkit, F. (2012). Abusive supervision and frontline employees' service performance. The Service Industries Journal, 32(5), pp. 683-698. doi:10.1080/02642069.2011.614338

Jensen, J. M., Van De Voorde, K. (2016). High performance at the expense of employee health? Reconciling the dark side of high performance work systems. In Ashkanasy, N. M., Bennett, R. J., \& Martinko, M. J. (Eds.), Understanding the high-performance workplace: The line between motivation and abuse (pp. 63-84). New York: Routledge.

Juárez, A., García, J., Camacho, A., Gómez, V., Vera, A., Fernández, M., \& García, I. (2011). Traducción y adaptación del MBI-GS en población latina. Documento no publicado.

Kaiser, H. F. (1960). The application of electronic computers to factor analysis. Educational and psychological measurement, 20(1), 141-151. doi:10.1177/001316446002000116

Khattak, A. J., Ahmad, W., Ahmad, A., \& Manzoor, H. (2015). The role of supervisor perceived intent as a moderator in the impact of abusive supervision on emotional exhaustion and turnover intention. Science International, 27(4).

Kline, R. B. (2011). Principles and practice of structural equation modeling (third edition). New York: The Guilford Press.

Krasikova, D. V., Green, S. G., \& LeBreton, J. M. (2013). Destructive leadership a theoretical review, integration, and future research agenda. Journal of Management, 39(5), 13081338. doi: $10.1177 / 0149206312471388$

Lian, H., Ferris, D. L., \& Brown, D. J. (2012a). Does taking the good with the bad make things worse? How abusive supervision and leader-member exchange interact to impact need satisfaction and organizational deviance. Organizational Behavior and Human Decision Processes, 117(1), 41-52. doi:10.1016/j.obhdp.2011.10.003

Lian, H., Ferris, D. L., \& Brown, D. J. (2012b). Does power distance exacerbate or mitigate the effects of abusive supervision? It depends on the outcome. Journal of Applied Psychology, 97(1), 107. doi:10.1037/a0024610

Liu, D., Liao, H., \& Loi, R. (2012). The dark side of leadership: A three-level investigation of the cascading effect of abusive supervision on employee creativity. Academy of Management Journal, 55(5), 1187-1212. doi:10.5465/amj.2010.0400

Liu, X. Y., \& Wang, J. (2013). Abusive supervision and organizational citizenship behaviour: Is supervisor-subordinate guanxi a mediator? The International Journal of Human Resource Management, 24(7), 14711489. doi:10.1080 / 09585192.2012.725082 
Little, R. J., \& Rubin, D. B. (2014). Statistical analysis with missing data. John Wiley \& Sons.

Mackey, J. D., Frieder, R. E., Brees, J. R., \& Martinko, M. J. (2015). Abusive supervision: A meta-analysis and empirical review. Journal of Management. doi: $10.1177 / 0149206315573997$

Martinko, M. J., Harvey, P., Brees, J. R., \& Mackey, J. (2013). A review of abusive supervision research. Journal of Organizational Behavior, 34(S1), S120-S137. doi:10.1002/job.1888

Meliá, J. L., Peiró, J. M. (1989). La medida de la satisfacción laboral en contextos organizacionales: El Cuestionario de Satisfacción S20/23 [The measurement of job satisfaction in organizational settings: The S20/23 Job Satisfaction Questionnaire]. Psicologemas, 5, 59-74.

Mitchell, M., \& Ambrose, M. (2007). Abusive supervision and workplace deviance and the moderating effects of negative reciprocity beliefs. Journal of Applied Psychology, 92(4), 1159-1168. doi:10.1037/0021-9010.92.4.1159

Moroni, L., \& Dabos, G. E. (2014). Comportamientos abusivos de baja intensidad en las organizaciones. Estudios Gerenciales, 30, 384-396. doi:10.1016/ j.estger.2014.05.003

Nader, M., \& Castro Solano, A. (2010). Teorías implícitas del liderazgo, LMX y bienestar laboral: Generalización de un modelo teórico. Revista de Psicología, 28(2). Disponible en: http://www.redalyc.org/html/970/9702103100 3/

Neider, L. (2010). The "dark" side of management. Charlotte, NC: Information Age Pub.

Pando, M., Aranda, C., Salazar, J., \& Torres, T. (2016). Prevalencia de violencia psicológica y acoso laboral en trabajadores de Iberoamérica. Enseñanza e Investigación en Psicología, 21(1), 39-45. Disponible en: www.redalyc.org/articulo.oa?id=29248180004

Park, H. S., Hoobler, J. M., Wu, J., \& Wilson, M. (2015, January). Abusive supervision, justice, power distance, and employee deviance: A meta-analysis. In Academy of Management Proceedings (Vol. 2015, No. 1, p. 12462).
Academy

of

Management. doi:10.5465/AMBPP.2015.73

Pedrosa, I., \& García-Cueto, E. (2016). Síndrome de Burnout en árbitros de élite: La liga de fútbol profesional española (LFP) a estudio. Revista Iberoamericana de Diagnóstico y Evaluación - e Avaliação Psicológica, 42(2), 59-68. doi:10.21865/RIDEP42_59

Pelletier, K.L. (2010). Leader toxicity: An empirical investigation of toxic behavior and rhetoric, Leadership, November, 6, 373-389. doi:10.1177/1742715010379308

Perilla-Toro, L. E., \& Gómez-Ortiz, V. (2017). Relación del estilo de liderazgo transformacional con la salud y el bienestar del empleado: El rol mediador de la confianza en el líder. Journal of Work and Organizational Psychology, 33, 95-108. doi:10.1016/j.rpto.2017.02.005

Priesemuth, M., Schminke, M., Ambrose, M., Folger, R. (2014). Abusive supervision climate: A multiple-mediation model of its impact on group outcomes. Academy of Management Journal, 57(5), 1513-1534. doi:10.5465/amj.2011.0237

Podsakoff, P. M., MacKenzie, S. B., Lee, J. Y., \& Podsakoff, N. P. (2003). Common method biases in behavioral research: A critical review of the literature and recommended remedies. Journal of Applied Psychology, 88, 879-903. doi:10.1037/0021-9010.88.5.879

Popa, M., Rotarescu, V. S., \& Sulea, C. (2013, July). Toxic leadership scale: A Romanian adaptation and validation. In Psychology \& Health (Vol. 28, pp. 291-291). Disponible en: http://pru.apio.ro/index.php/prujournal/article/ view/107/110

Pradhan, S., \& Jena, L. K. (2017). Effect of abusive supervision on employee's intention to quit and the neutralizing role of meaningful work in Indian IT organizations. International Journal of Organizational Analysis, (justaccepted). doi:10.1108/IJOA-01-2017-1107

Pyc, L. S., Meltzer, D. P., \& Liu, C. (2017). Ineffective leadership and employees' negative outcomes: The mediating effect of anxiety and depression. International Journal of Stress Management, 24(2), 196. doi:10.1037/str0000030 
Reed, G. E. (2004). Toxic leadership. Military Review, 84(4), 67-71. Disponible en: cgsc.contentdm.oclc.org/cdm/ref/collection/p1 24201coll1/id/182

Rodríguez-Muñoz, A. (2011). Acoso psicológico en el trabajo: Revisión de la literatura y nuevas líneas de investigación. Medicina y Seguridad del trabajo, 57(1), 20-34. Disponible en: www.redalyc.org/pdf/292/29228336011.pdf

Rosseel, Y. (2012). Lavaan: An R package for structural equation modeling and more. Version 0.5-12 (BETA). Journal of statistical software, $\quad 48(2), \quad 1-36$. http://www.jstatsoft.org/v48/i02/.

Rupp, D. E. (2011). An employee-centered model of organizational justice and social responsibility. Organizational Psychology Review, 1, 72-94. doi:10.1177/ 2041386610376255

Ryan, R. M., \& Deci, E. L. (2017). Selfdetermination theory: Basic psychological needs in motivation, development, and wellness. New York: The Guilford Press.

Schaufeli, W. B., \& Bakker, A. B. (2003). UWES-Utrecht work engagement scale: Test manual. Unpublished Manuscript: Department of Psychology, Utrecht University, $8 . \quad$ Disponible en: http://www.wilmarschaufeli.nl/publications/S chaufeli/Test\%20Manuals/

Test_manual_UWES_Espanol.pdf

Scheuer, M. L., Burton, J. P., Barber, L. K., Finkelstein, L. M., \& Parker, C. P. (2016). Linking abusive supervision to employee engagement and exhaustion. Organization Management Journal, 13(3), 138-147. doi:10.1080/15416518.2016.1214063

Schmidt, A. A. (2008). Development and validation of the toxic leadership scale. ProQuest. Disponible en: http://hdl.handle.net/1903/8176

Shadish, W. R., Cook, T. D., \& Campbell, D. T. (2001). Experimental and quasiexperimental designs for generalized causal inference. Boston, MA: Houghton Mifflin Company.

Shirom, A. (2010). Feeling energetic at work: On vigor's antecedents. In Leiter, M. P., \& Bakker, A. B. (Eds.), Work engagement: A handbook of essential theory and research (pp. 69-84). Psychology press.
Tepper, B. J. (2000). Consequences of abusive supervision. Academy of Management Journal, 43, 178-190. doi:10.2307/1556375

Tepper, B. J., Duffy, M. K., Hoobler, J., \& Ensley, M. D. (2004). Moderators of the relationships between coworkers' organizational citizenship behavior and fellow employees' attitudes. Journal of Applied Psychology, 89(3), 455. doi:10.1037/0021-9010.89.3.455

Tepper, B. J. (2007). Abusive supervision in work organizations: Review, synthesis, and research agenda. Journal of Management, 33(3), 261289. doi:10.1177/0149206307300812

Tepper, B. J., \& Almeda, M. (2012). Negative exchanges with supervisors. In de Tormes Eby, L., \& Allen, T.D. (Eds.), Personal relationships: The effect on employee attitudes, behavior, and well-being (pp. 6793). New York: Routledge.

Thoroughgood, C., Tate, B., Sawyer, K., \& Jacobs, R. (2012). Bad to the bone: Empirically defining and measuring destructive leader behavior. Journal of Leadership \& Organizational Studies, 19, 230-255. doi:10.1177/ 1548051811436327

Topa, G., Depolo, M., \& Morales, J. F. (2007). Acoso laboral: Meta-análisis y modelo integrador de sus antecedentes y consecuencias. Psicothema, 19, 88-94. Disponible en: http:// www.redalyc.org/articulo.oa?id=72719113

Toro, J. P., \& Gómez-Rubio, C. (2016). Factores facilitadores de la violencia laboral: Una revisión de la evidencia científica en América Latina. Ciencia \& trabajo, 18(56), 110-116. Disponible en: http://www.scielo.cl/pdf/cyt/v18n56/art06.pdf

Wei, F., \& Si, S. (2013). Tit for tat? Abusive supervision and counterproductive work behaviors: The moderating effects of locus of control and perceived mobility. Asia Pacific Journal of Management, 30(1), 281-296. doi:10.1007/s10490-011-9251y

Whicker, M. L. (1996). Toxic leaders: When organizations go bad. Westport, CT: Quorum Books.

Wu, S., \& Cao, K. (2015). Abusive supervision and work-family conflict: The mediating role of emotional exhaustion. Journal of Human 
Resource and Sustainability Studies, 3(04), 171. doi:10.4236/jhrss.2015.34023

Xu, S., Martinez, L. R., Van Hoof, H., Tews, M., Torres, L., \& Farfan, K. (2015). The impact of abusive supervision and co-worker support on hospitality and tourism student employees' turnover intentions in Ecuador. Current Issues in Tourism, 1-16.

doi:10.1080/13683500.2015.1076771

Zellars, K. L., Tepper, B. J., \& Duffy, M. K. (2002). Abusive supervision and subordinates' organizational citizenship behavior. Journal of Applied Psychology, 87(6), 1068-1076. doi:10.1037/0021-9010.87.6.1068

Zhang, Y., \& Bednall, T. C. (2015). Antecedents of abusive supervision: A meta-analytic review. Journal of Business Ethics, 1-17. doi:10.1007/s10551-015-2657-6

Zwick, W. R., \& Velicer, W. F. (1986). Comparison of five rules for determining the number of components to retain. Psychological Bulletin, 99(3). doi:10.1037/0033-2909.99.3.432 